\title{
Die Geschwindigkeit des Kathodenstrahls eines Quecksilberdampf-Niederdruckbogens
}

\author{
Von Heinz Nöske und Er win Schmidt \\ Aus dem Schaltwerk der Siemens-Schuckertwerke A.-G., Berlin-Siemensstadt \\ (Z. Naturforschg. 7a, 677-682 [1952]; eingegangen am 6. Juni 1952)
}

\begin{abstract}
Der Metallkathode einer Niederdruck-Bogenentladung entweicht ein Dampfstrahl. An einer Hg-Kathode wird die Geschwindigkeit dieses Dampfstrahls dadurch gemessen, daß sein zeitliches Eintreffen in verschiedenen Entfernungen von der Kathode mit Hilfe von Sonden festgestellt wird. Die Bestimmung der Laufzeiten bei verschiedenen $\mathrm{Hg}-$ Dampfdrucken ergibt, daß unterhalb eines Druckes, für den die freie Weglänge in die Größenordnung der Gefäßdimensionen fällt, die Dampfstrahlgeschwindigkeit einen oberen Grenzwert von $1,3 \cdot 10^{5} \mathrm{~cm} / \mathrm{sec}$ für die wahrscheinlichste Geschwindigkeit annimmt, der sich als unabhängig davon erweist, in welcher Entfernung von der Kathode die Strahlgeschwindigkeit bestimmt wird.
\end{abstract}

$\mathrm{V}$ on der Kathode eines Niederdruckbogens geht ein aus Kathodenmaterial bestehender Dampfstrahl aus. Tanberg ${ }^{1}$ bestimmte 1930 für einen Niederdruckbogen mit Cu-Kathode durch Messung des Dampfstrahlimpulses sowie der in der Zeiteinheit verdampften $\mathrm{Cu}$-Menge die Strahlgeschwindig-

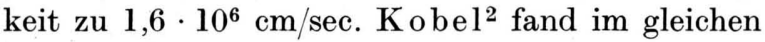
Jahr für den Fall eines Hg-Niederdruckbogens nach einer ähnlichen Meßmethode Strahlgeschwindigkeiten von derselben Größenordnung. In neuerer Zeit bestimmte Haynes ${ }^{3}$ die Geschwindigkeit des von der $\mathrm{Hg}$-Kathode einer Funkenentladung in 900 Torr Wasserstoff ausgehenden Dampfstrahls zu $1,9 \cdot 10^{5} \mathrm{~cm} / \mathrm{sec}$. Nach Haynes ist der Dampfstrahl unabhängig von der Entladungsbahn senkrecht zur Kathodenoberfläche gerichtet. Während die früheren Messungen an Niederdruckbögen um etwa eine Zehnerpotenz höhere Geschwindigkeiten als die an Funkenentladungen ergaben, sollte nach Haynes der Mechanismus der Dampfstrahlbildung und damit auch die Geschwindigkeit in beiden Fällen gleich sein.

Nach einer Anregung von Steen beck führte der eine von uns (E. S.) 1943 neue Untersuchungen über die Strahlgeschwindigkeit im Hg-Niederdruckbogen durch, deren Resultat mit der späteren Messung von Haynes an Funkenentladungẹ in größenordnungsmäßiger Übereinstimmung war. Da sowohl die Meßapparatur wie auch alle Unterlagen durch den Krieg verlorengingen, wurden diese Versuche jetzt wiederholt ${ }^{4}$.

${ }^{1}$ R. Tanberg, Physic. Rev. 35, 1080 [1930].

2 E. Kobel, Physic. Rev. 36, 1936 [1930].

3 J. R. Haynes, Physic. Rev. 73, 891 [1948], s. dort auch weitere Literaturangaben.

\section{Versuchsanordnung und Meßverfahren}

Das Versuchsgefä $\beta$ (Abb. 1) besteht im wesentlichen aus einem kreiszylindrischen, sorgfältig evakuierten Glasgefäß mit Hg-Kathode, Molybdän-Brennfleckfixierung und Graphitanode. In ihm brennt während der ganzen Versuchsdauer ein Gleichstrombogen von einigen A. Dem Gleichstrom werden hohe kurze Stromimpulse überlagert, die eine entsprechende Trägerkonzentrationsänderung in dem vom Brennfleck in das Rohr strömenden ,Dampfstrahl bewirken. Längs der

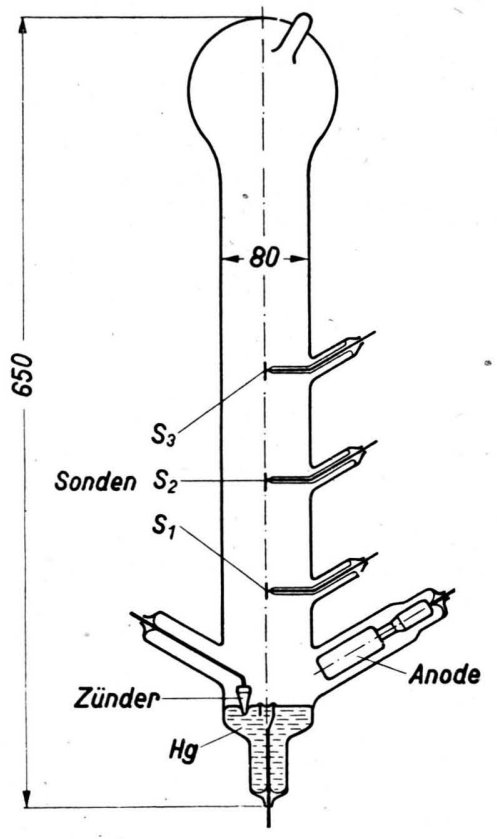

Abb. 1. Versuchsgefä $ß$.

${ }^{4}$ H. Nöske, Dipl.-Arbeit, Techn. Univ. Berlin 1951; E. Schmidt, Referat Gasentladungstagung Bonn am 19.7. 1952 . 
Achse des Versuchsgefäßes sind, in gleichen Abständen von der Kathode an gerechnet, ebene kreisscheibenförmige Sonden $\mathbf{S}_{1}, \mathbf{S}_{2}, \mathbf{S}_{3}$ von $1 \mathrm{~cm}$ Durchmesser angeordnet, derart, daß ihre Flächen in der Rohrachse liegen und der Dampfstrahl somit an beiden Sondenflächen vorbeiströmen kann. Erreicht die durch den Stromimpuls in Kathodennähe erzeugte und vom Dampfstrahl mitgeführte Trägerwolke eine der Sonden, so ruft sie dort eine Erhöhung des Sondenstromes hervor, die oszillographisch gemessen wird. Aus dem zeitlichen Abstand der Strommaxima an den einzelnen Sonden kann man auf die Geschwindigkeit des Dampfstrahls schließen.

Abb. 2 zeigt schematisch die verwendete Schaltung. Als Spannungsquelle für die Stromimpulse dient eine mit $50 \mathrm{~Hz}$ periodische Kondensatorentladung von 20 bis 200 A und $1-10 \mu$ sec Dauer. Die Sonden liegen über Vorwiderstände von $70-200 \mathrm{k} \Omega$ an einer negativen Gleichspannung von $60 \mathrm{~V}$; gemessen wurde also der positive Ionenstrom. Die seinen Schwankungen proportionalen Spannungsänderungen am Sondenvorwiderstand überträgt ein Wechselstromverstärker auf das eine Plattenpaar einer Braunschen Röhre. Dadurch, daß die Zeitablenkspannung des Oszillographen mit den Stromimpulsen synchronisiert wird, erscheint auf seinem Schirm ein stehendes Bild des zeitlichen Sondenstromverlaufs der jeweils angeschlossenen Sonde. Das Versuchsgefä $\beta$ befindet sich vollständig in einem Wasserbad mit geregelter Temperatur, die den Hg-Dampfdruck im Gefäß bestimmt. Zur Zündung der Entladung dient ein Ignitron-Zündstift.

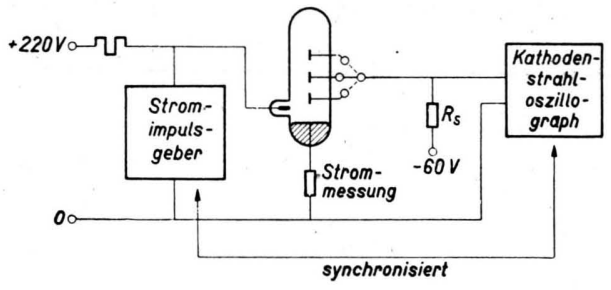

Abb. 2. Blockschema der Schaltung.

\section{Meßergebnisse}

In Abb. 3 sind die relativen Sondenströme als Funktion der Zeit für einen Gasdruck von etwa $10^{-3}$ Torr aufgetragen, wobei als Nullpunkt der Zeitachse der Beginn des in Abb. 4 dargestellten Stromimpulses angenommen wurde. Letzterer wurde aus dem Spannungsabfall am Meßwiderstand von Abb. 2 oszillographisch ermittelt. Das Verhältnis der maximalen Sondenströme der ersten zur zweiten und dritten Sonde beträgt in diesem Falle etwa 20:1,2:0,05. Eine Abhängigkeit der Laufzeit des Dampfstrahlimpulses von der Dauer und Höhe des Stromimpulses innerhalb der bereits angegebenen Grenzen konnte in Übereinstimmung mit Haynes nicht festgestellt werden.

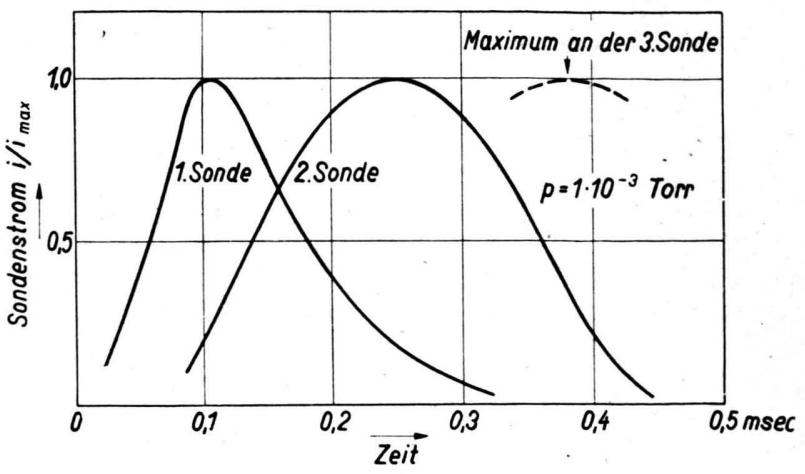

Abb. 3. Sondenströme als Funktion der Zeit.

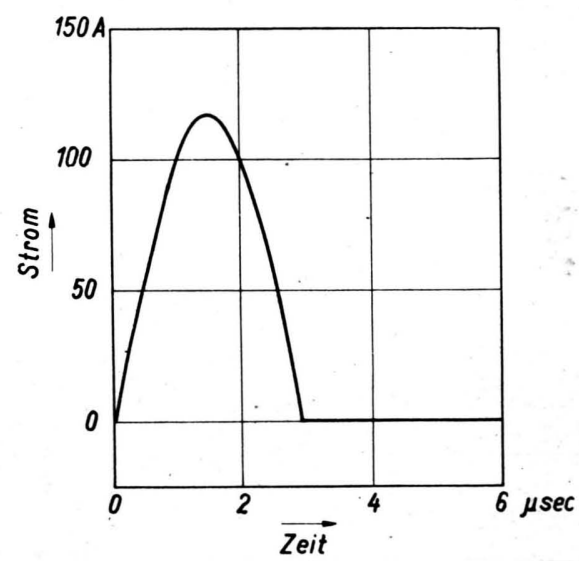

Abb. 4. Zeitlicher Stromimpulsverlauf.

Das Ergebnis unserer Laufzeitmessung in Abhängigkeit vom Hg-Dampfdruck ist in Abb. 5 dargestellt. Die Kurven I beziehen sich auf die Laufzeit des Dampfstrahls zwischen Kathode und der $9,6 \mathrm{~cm}$ von ihr entfernten ersten Sonde, die Kurve II dagegen auf die Laufzeit zwischen der ersten und der 10,7 cm von ihr entfernten zweiten Sonde, und zwar geben die Kurven Ib, IIb die aus der zeitlichen Verschiebung der Maxima der Sondenströme $i_{\max }$ berechneten Werte wieder, während Ia, II a diejenigen bezeichnen, für welche die Bewegung der Plasmafront zugrunde gelegt wurde. Zur Kennzeichnung der Plasmafront dient der Sondenstrom $i_{\mathrm{s}}=i_{\max } / 2$. Die Messungen an der dritten Sonde wurden nicht mehr ausgewertet, da bei ihr die Sondenstromänderungen nur noch für hohe Stromimpulse gerade feststellbar waren. Man erkennt, daß die Laufzeit mit abnehmendem Druck sinkt und einem konstanten Wert zustrebt, der offenbar dadurch gegeben ist, daß hier die freie Weglänge be- 
reits größer als der Sondenabstand ist (für $p=2 \cdot 10^{-4}$ Torr und $0^{0} \mathrm{C}: \lambda \approx 8 \mathrm{~cm}$ ). Bei der Auswertung unserer Messung wurden die Laufzeiten bis zur ersten Sonde ebenso wie die von dieser zur zweiten Sonde mit gleichem Gewicht unter Berücksichtigung der genau ausgemessenen Abstände herangezogen.

Für die maximale, durch das Grundgas nicht mehr beeinflußte Dampfstrahlgeschwindigkeit (= Sondenabstand/Laufzeit) ergibt sich bei Bezugnahme der Zeitmessung auf jenen Zeitpunkt, zu dem

1. der Sondenstrom $i_{\mathrm{s}}$ seinen Maximalwert erreicht hat:

$$
v_{1}=1,5 \cdot 10^{5} \mathrm{~cm} / \mathrm{sec},
$$

2. der Sondenstrom $i_{\mathrm{s}}=i_{\max } / 2$ beträgt:

$$
v_{2}=2,8 \cdot 10^{5} \mathrm{~cm} / \mathrm{sec} \text {. }
$$

Die von Hay nes gemessene Geschwindigkeit von $1,9 \cdot 10^{5} \mathrm{~cm} / \mathrm{sec}$ liegt etwa in der Mitte zwischen diesen beiden Werten.

In Abb. 6 sind die Laufzeiten der Dampfstrahlmaxima zwischen Kathode und erster Sonde in $\mathrm{Ab}$ hängigkeit von Gleichstrom aufgetragen. Parameter der Kurve ist der Dampfdruck. Wie man aus dieser Darstellung erkennt, nimmt oberhalb eines Druckes von $5,7 \cdot 10^{-4}$ Torr die Laufzeit mit steigendem

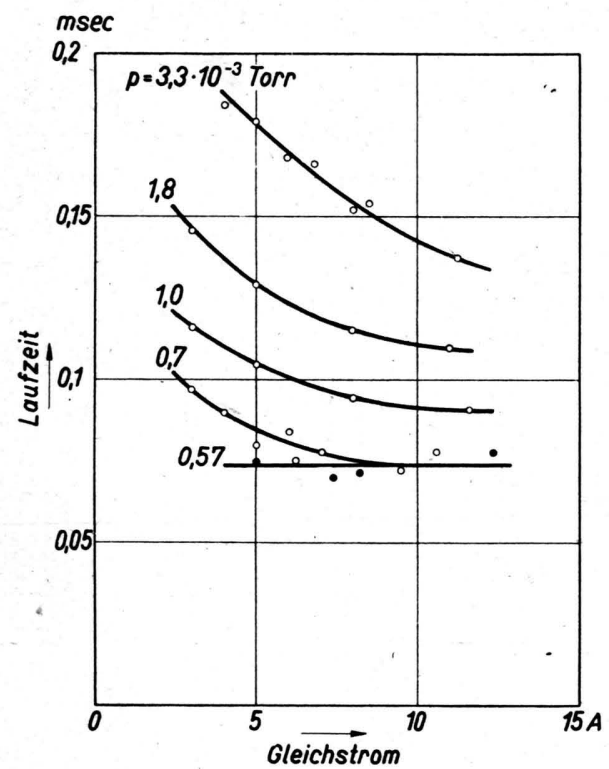

Abb. 6. Laufzeit des Dampfstrahlmaximums zwischen Kathode und der $9,6 \mathrm{~cm}$ von ihr entfernten 1. Sonde in Abhängigkeit vom Gleichstrom bei verschiedenen Drucken.

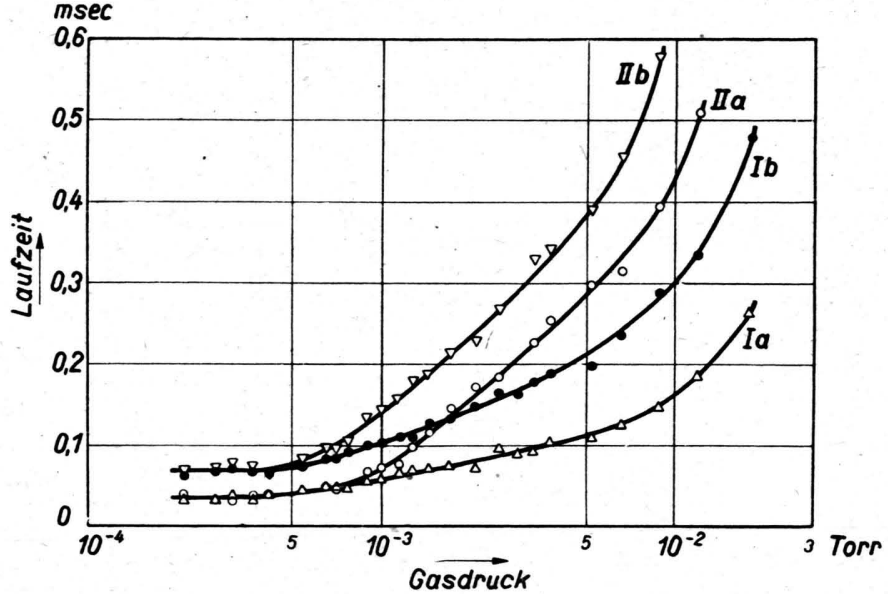

Abb. 5. Laufzeit des Dampfstrahls in Abhängigkeit vom Gasdruck.

Ia Front des Strahls $\left(i_{\mathrm{s}}=i_{\max } / 2\right) \quad$ zwischen Kathode und der Ib Max. , , , $\quad\left(i_{\mathrm{s}}=i_{\max }\right) \quad\left\{\begin{array}{l}9,6 \mathrm{~cm} \\ 1 . \text { Sonde }\end{array}\right.$

IIa Front ,, , $\quad\left(i_{\mathrm{s}}=i_{\max } / 2\right) \quad$ (zwischen der 1. und der IIb Max. , , , , $\quad\left(i_{\mathrm{s}}=i_{\max }\right) \quad\{10,7 \mathrm{~cm}$ von ihr entfernten

Gleichstrom ab. Die Ursache hierfür ist wohl darin zu suchen, daß ein höherer Gleichstrom eine verstärkte Ausbildung der stationären Dampfströmung bewirkt, so daß die durch den Impuls zusätzlich erzeugte Strömung weniger durch das Grundgas behindert wird.

Eine nennenswerte Erhöhung des Kathodenfalls durch den Stromimpuls konnte nicht festgestellt werden. Damit entfällt die Möglichkeit, daß die von uns zur Messung benutzten positiven Ionen als Ionisierungseffekt schneller Elektronen entstehen und so den Dampfstrahl vortäuschen könnten.

\section{Diskussion der Meßergebnisse}

Obgleich die von uns verwendeten Sonden zu große Oberflächen besitzen, als daß für sie die Langmuirsche Sondentheorie strenge Gültigkeit hätte, läßt sich zeigen, daß es sich bei der von uns zur Bestimmung der Dampfstrahlgeschwindigkeit ausgemessenen, auf dem Leuchtschirm des Elektronenstrahlrohres erscheinenden Kurvenform tatsächlich um das Abbild des zeitlichen Verlaufs der Trägerkonzentration am Ort der Sonde handelt.

$\mathrm{Zu}$ diesem Zweck könnte man im Prinzip eine Sondenkennlinie innerhalb eines so kurzen Zeitintervalles aufnehmen, daß sich die Trägerkonzentration während der Meßzeit am Ort der Sonde nicht nennenswert ändert. Statt dessen haben wir 
an der zweiten Sonde $S_{2}$ für den Zeitpunkt des Maximums der Trägerkonzentration eine derartige dynamische Kennlinie auf folgendeWeise gewonnen : Beobachtet wird die Höhe des Sondenstrommaximums in Abhängigkeit von der an der Sonde liegenden Spannung, die sich aus der angelegten Gleichspannung und dem Spannungsabfall am Sondenwiderstand ergibt. Durch Variation von Größe und Vorzeichen der Vorspannung sowie des Widerstandes ist es möglich, die Sondenkennlinie punktweise zu ermitteln. Sie zeigt in unserem Falle den üblichen Verlauf; aus dem Elektronensättigungsstrom ergab sich eine Trägerdichtezunahme von $8 \cdot 10^{8}$ auf $1 \cdot 10^{9} \mathrm{~cm}^{-3}$. Hierbei betrugen $J=3 \mathrm{~A}, p=1 \cdot 10^{-3}$ Torr, Stromstoß $=3 \cdot 10^{-4}$ Asec.

Grundsätzlich wäre es auch möglich gewesen, die Meßmethode auf die Untersuchung des zeitlichen Verlaufs des Elektronensättigungsstromes an den Sonden einzurichten. Wir haben jedoch die Messung des Ionensättigungsstromes vorgezogen, da dessen Spannungsbereich nicht durch den Übergang in eine selbständige Entladung eingeschränkt wird.

Als maximale Einstellzeit des Sondenstromes auf eine plötzliche Trägerkonzentrationsänderung darf man diejenige Laufzeit annehmen, die ein Plasmaion benötigt, um von der Grenze zwischen Plasma und positiver Raumladungsschicht zur Sondenoberfläche zu gelangen. Mit Benutzung der Lang muirschen Raumladungsformel ergibt die Rechnung in unserem Fall $\tau_{+}=5 \cdot 10^{-6}$ sec. Sie ist also gegen jene Zeit von $1 \cdot 10^{-4}$ sec, in welcher der Sondenstrom vom halben auf das volle Maximum steigt, $\mathrm{zu}$ vernachlässigen.

Bei unserer Messung der Dampfstrahlgeschwindigkeit wurde bisher vorausgesetzt, daß z. B. das Dichtemaximum des Dampfstrahls die Sonde zu dem Zeitpunkt erreicht, zu dem diese maximalen Strom führt. Daß diese Annahme nicht zutrifft, ergibt sich aus folgender Überlegung:

Wenn man von der Konzentrationsabnahme mit der Zeit in radialer Richtung des Versuchsrohres, z. B. durch ambipolare Diffusion, absieht, läßt sich unter der von $\mathrm{Haynes}{ }^{3}$ bestätigten Annahme, daß der Dampfstrahl senkrecht zur Kathodenoberfläche gerichtet ist und bei Kenntnis der je Zeit- und Flächeneinheit von der Kathode ausgehenden Anzahl $N$ von Dampfteilchen sowie deren Geschwindigkeitsverteilung $\varphi(v)$ der zeitliche Dampfdichteverlauf $n(z, t)$ an einem beliebigen Ort $z$ der Achse des Versuchsrohres als eindimensionales Problem leicht berechnen. Für einen Zeitpunkt $t$ ergibt die Rechnung

$$
n(z, t)=\frac{1}{z} \int_{0}^{t} N(\vartheta) \frac{\dot{z}}{t-\vartheta} \varphi\left(\frac{z}{t-\vartheta}\right) \mathrm{d} \vartheta .
$$

Nimmt man insbesondere für $\varphi(v)$ Maxwell-Geschwindigkeitsverteilung an, so wird

$$
\begin{gathered}
n(z, t)=\frac{A}{z} \int_{0}^{t} N(\vartheta)\left(\frac{z}{t-\vartheta}\right)^{3} e^{-b\left(\frac{z}{t-\vartheta}\right)^{2}} \mathrm{~d} \vartheta \\
\operatorname{mit}\left\{\begin{array}{l}
A=\frac{4}{\sqrt{\pi}}\left(\frac{m}{2 k T}\right)^{3 / 2}, \\
b=\frac{m}{2 k T} .
\end{array}\right.
\end{gathered}
$$

Für die bei unserer Messung erfüllte Voraussetzung, daß die Dauer $\vartheta_{0}$ des Stromimpulses klein gegen die Laufzeit $t$ des Strahls bis zur Sonde am Ort $z$ ist, d. h. $\vartheta_{0} \ll t$, und unter der bereits von Tanberg ${ }^{1}$ bestätigten Voraussetzung, daß die Anzahl $N$ der je Zeiteinheit vom Kathodengebiet ausgehenden Dampfteilchen proportional der jeweiligen Stromstärke $i$ ist, erweist $\operatorname{sich} n(z, t)$ als weitgehend unabhängig vom zeitlichen Vèrlauf und der Dauer des Stromimpulses, soweit nur $\int_{0}^{\vartheta_{0}} i(\vartheta) \mathrm{d} \vartheta$ gleich bleibt, was auch bei der Messung durch Variation von $i(\vartheta)$ bestätigt werden konnte. Für die folgenden Überlegungen sollen diese Voraussetzungen immer als erfüllt gelten.

Wir fragen nach der Zeit $t_{\text {max }}$, zu der für ein bestimmtes $z n(t)$ ein Maximum wird, oder mit anderen Worten ausgedrückt, zu der unsere Sonde maximalen Strom führt. Aus (2) ergibt sich hierfür durch Nullsetzen der ersten partiellen Ableitung nach $t$ für den speziellen Fall einer Maxwell-Geschwindigkeitsverteilung für

$$
t_{\max }=z \sqrt{\frac{2}{3} b}=z \sqrt{\frac{m}{3 k T}} .
$$

Indem wir diesen Wert in den Ausdruck einführen, der sich aus (2) durch Nullsetzen der ersten partiellen Ableitung nach $z$ ergibt, erhalten wir den Ort $z_{\text {max }}$, an dem sich das Dichtemaximum des Dampfstrahls zur Zeit $t_{\max }$ befindet:

$$
z_{\max }=\frac{t_{\max }}{\sqrt{b}}=t_{\max } \sqrt{\frac{2 k T}{m}} .
$$

Dem entspricht bei angenommener Maxwell-Geschwindigkeitsverteilung die wahrscheinlichste Geschwindigkeit 


$$
v_{\mathrm{w}}=\frac{z_{\max }}{t_{\max }}=\sqrt{\frac{2 k T}{m}}
$$

während aus (3) für die durch unsere Messung bestimmte Strahlgeschwindigkeit

$$
V=\frac{z}{\iota_{\max }}=\sqrt{\frac{3 k T}{m}} \sqrt{\frac{3}{2}} v_{\mathrm{w}}=\sqrt{\overline{v^{2}}}
$$

folgt, d. h. unsere gemessene Geschwindigkeit wäre identisch mit der Wurzel aus dem mittleren Geschwindigkeitsquadrat. Wendet man diese Überlegungen auf die von uns gemessenen SondenstromZeitkurven $n(z, t)$ ohne Voraussetzung einer Maxwell-Geschwindigkeitsverteilung an, so ergibt sich folgendes:

Da $n(z, t)$ sich als unabhängig von $N(\vartheta)$ erwiesen hat, nehmen wir für $N(\vartheta)$ willkürlich einen Rechteckverlauf an, d. h.

$$
N(\vartheta)= \begin{cases}0 & \text { für } \vartheta<0 \text { und } \vartheta>\vartheta_{0}, \\ N=\text { const. für } 0 \leqq \vartheta \leqq \vartheta_{0} .\end{cases}
$$

Dann wird (1)

$$
n(z, t)=N \int_{0}^{t} \varphi\left(\frac{z}{t-\vartheta}\right) \frac{d \vartheta}{(t-\vartheta)} \approx N \varphi\left(\frac{z}{t}\right) \frac{\vartheta_{0}}{t},
$$

d.h. für die Geschwindigkeitsverteilungsfunktion $\varphi(v)$ gilt

$$
\varphi(v)=\varphi\left(\frac{z}{t}\right)=\frac{n(z, t) t}{N \vartheta_{0}} \sim i_{\mathrm{s}} t .
$$

Führt man das in unserem Falle durch, so ergibt sich für die wahrscheinlichste Geschwindigkeit, das ist jene, für die $\varphi(v)$ ein Maximum hat,

$$
v_{\mathrm{w}}=1,3 \cdot 10^{5} \mathrm{~cm} / \mathrm{sec}
$$

und für die Geschwindigkeit jener Teilchen, deren Anzahl gleich der halben sich mit maximaler Geschwindigkeit bewegender Teilchen ist,

$$
v_{\mathrm{w} / 2}=1,9 \cdot 10^{5} \mathrm{~cm} / \mathrm{sec},
$$

was mit dem von Haynes ${ }^{3}$ gemessenen Wert der Frontgeschwindigkeit übereinstimmt, der allerdings die Frage der Geschwindigkeitsverteilung nicht erörtert. Es wäre prinzipiell möglich, auf diese Weise aus den Sondenstrom-Zeitkurven die Geschwindigkeitsverteilung der Ionen im Dampfstrahl anzugeben. Wir haben darauf verzichtet, da wir einerseits die Konzentrationsabnahme durch ambipolare

\footnotetext{
${ }^{5}$ W. Koch, Z. techn. Physik 17, 446 [1936].

6 Nach brieflicher Mitteilung durch Herrn J. v. Is sendorff handelt es sich vielleicht hier auch um einen Effekt, der durch ambipolare Diffusion hervorgerufen wird, die mit höherer Geschwindigkeit dem Dampf-
}

Diffusion in radialer Richtung bei unserer Rechnung außer acht gelassen haben, und andererseits der glatte Verlauf der Sondenstromkurven bei den niedrigsten Drucken durch das Auftreten eines Nebenmaximums im ansteigenden Kurvenast gestört wurde. Eine eindeutige Erklärung für das Auftreten des Nebenmaximums wurde nicht gefunden, hierzu wären wohl Messungen mit verschiedenen Sondenformen notwendig. Nach unserer Meinung käme als Ursache der durch die zeitliche Ionenkonzentrationsschwankung beim Eintreffen des Strahlkopfes am Ort der Sonden nach $\mathrm{Koch}^{5}$ bedingte dynamische, dem Ionenstrom entgegengesetzte Sondenstrom in Frage, auch die verschiedene Laufzeit der Ionen und Elektronen des Dampfstrahlimpulses durch das Raumladungsgebiet der Sonden könnte die Erscheinung eines Doppelmaximums hervorrufen ${ }^{6}$.

Wir glauben trotz dieser Erscheinung annehmen zu dürfen, daß die von uns gemessene Geschwindigkeit des Dampfstrahldichtemaximums nicht durch das Auftreten des zweiten Maximums gefälscht ist, da sich die störenden Vorgänge ausschließlich im mittleren Teil des ansteigenden Astes der Sondenstrom-Zeitkurven abspielen. Außerdem treten die Störeffekte erst bei den niedrigsten von uns zur Messung benutzten Dampfdrucken in erheblicher Größe auf, nachdem bereits das druckunabhängige Gebiet der Laufzeiten in Abb. 5 erreicht ist. Aus diesem Grunde, nämlich aus der Kontinuität der gemessenen Laufzeiten in Abb. 5, dürfte auch die von uns gemessene Frontgeschwindigkeit des Dampfstrahls mit genügender Genauigkeit bestimmt worden sein, zumal das Existieren einer Grenzgeschwindigkeit bei Erreichen eines Druckes, bei welchem die freie Weglänge in die Größenordnung der Gefäßdimensionen fällt, aus rein physikalischen Gründen gefolgert werden muß. Aus der von uns gemessenen wahrscheinlichsten Geschwindigkeit folgt, wenn man als ihre Ursache die thermische Erzeugung des Dampfstrahls ansieht, eine Temperatur von $20000^{\circ} \mathrm{K}$, während Tanberg ${ }^{1}$ für seine Messungen am Cu-Bogen $5 \cdot 10^{5}{ }^{0} \mathrm{~K}$ fand. Diese errechnete hohe Temperatur war Anlaß zu zahlreichen Diskussionen ${ }^{3}$ dieser von Tanberg zuerst angegebenen Ursache des Dampfstrahls. Weizel, Rompe und Schön ${ }^{7}$ haben die von Slepian vorgeschlagene Annahme, daß der

strahl vorauseilt, weil die Konzentration der Ladungsträger im Kondensraum nach oben hin abnimmt.

${ }^{7}$ W. Weizel u. R. Rompe, Theorie elektrischer Lichtbögen und Funken, Joh. A. Barth-Verlag, Leipzig 1949 . 
Strom an der Kathode eines Hochdruckbogens von Ionen gedeckt wird, durchgerechnet und nehmen dicht vor der Kathode ein thermisch ionisiertes Gebiet hoher Temperatur an. Die von Haynes ${ }^{3}$ beobachtete Richtwirkung im Dampfstrahl ist jedoch durch diese Theorie nicht zu verstehen. Interessant ist die Tatsache, daß van Voorhis und Compton $^{8-10}$ für $\mathrm{He}, \mathrm{Ne}$ und $\mathrm{A}$ und Larché ${ }^{11}$ für einen Edelgashochdruckbogen einen von den nicht verdampfenden Kathoden ausgehenden Gasstrahl finden. Demnach spricht vieles dafür, daß das Gebiet

${ }^{8}$ Vanyoorhis u. K. J. Compton, Physic. Rev. 37, 1596 [1931].

9 E. S. Lamar, Physic. Rev. 37, 842 [1931]; Physic. Rev. 43, 169 [1933].

10 K. J. Compton u. E. S. Lamar, Physic. Rev. 44, 338 [1933].

11 K. Larché, Lichttechn. 2, 41 [1950].

${ }^{12}$ L. B. Loeb, Fundamental Processes of Electrical Discharge in Gases, J. Wiley \& Sons, Inc., London, New York [1939]. der Strahlerzeugung im Kathodenfall selbst zu suchen ist und daß es sich bei diesen schnellen Dampfteilchen im Sinne von Compton ${ }^{10}$, Loeb $^{12}$ und Haynes ${ }^{3}$ um ursprünglich auf die Kathode zufliegende Ionen handelt, die bei ihrer Neutralisation an der Kathode teilweise reflektiert werden. Zumindest größenordnungsmäßig läßt sich die von uns gemessene Geschwindigkeit auf diese Weise erklären; denn bei einem Kathodenfall von rund $6 \mathrm{~V}$ liegt die Auftreffgeschwindigkeit von Hg-Ionen bei etwa $2,5 \cdot 10^{5} \mathrm{~cm} / \mathrm{sec}^{13}$.

13 Herr J. v. Isse nd orff weist uns freundlicherweise darauf hin, daß der von uns untersuchte Dampfstrahl möglicherweise nicht im Brennfleck selbst, sondern ähnlich wie bei seinen Messungen der verdampften Hg-Menge (Physik. Z. 19, 857 [1928]) von der durch den Erregerbogen aufgeheizten $\mathrm{Hg}$-Oberfläche ausgeht. Dagegen sprechen jedoch die thermisch unverständlich hohen Geschwindigkeiten und besonders die von Haynes ${ }^{3}$ nachgewiesene ausgesprochene Richtwirkung.

\title{
Über die Entmischung von Lösungen des Polymethacrylesters II $^{1}$
}

\author{
Von E. Jenckel und J. Delahaye \\ Aus dem Institut für theoretische Hüttenkunde und physikalische Chemie \\ der Techn. Hochschule Aachen \\ (Z. Naturforschg. 7a, 682-692 [1952]; eingegangen am 19. April 1952)
}

\begin{abstract}
Es wurden die Löslichkeitskurven einer Anzahl von Fraktionen des Polymethacrylsäuremethylesters mit Polymerisationsgraden von 500 bis 30000 in $p$-Cymol, $n$-Propanol und $n$-Butanol bestimmt.

Es wurden die Koordinaten des kritischen Lösůngspunktes thermodynamisch berechnet. Hierbei bewährt sich vorzüglich ein Ansatz für die Mischungsentropie athermischer Lösungen von Miller in Verbindung mit einem quadratischen Ausdruck $\mu x_{2}^{2}$ für die Lösungswärme und die zusätzliche Entropie der irregulären Lösung, in dem $\mu=\alpha+\beta / T$ zu setzen ist.
\end{abstract}

$\mathrm{F}$ ür binäre Lösungen aus hochmolekularen und niedrigmolekularen Stoffen, die sich beim Abkühlen in zwei flüssige Phasen entmischen, ist es kennzeichnend, daß die kritische Zusammensetzung sehr weit zur Seite des reinen Lösungsmittels verschoben ist. Die vorliegende Arbeit untersucht im Anschluß an eine frühere ${ }^{1}$ Mitteilung solche Löslichkeitskurven an Polymethacrylester. In der früheren Arbeit wurde versucht, die Koordinaten des kritischen Lösungspunktes thermodynamisch zu berechnen. Diese thermodynamische Berechnung ist ein vorzügliches Kriterium dafür, ob die verwendeten Entropie- und Enthalpieformeln richtig sind

${ }^{1}$ Vgl. I: E. Jenckel u. K. Gorke, Z. Naturforschg. 5a, $556[1950]$. oder nicht. Die damals verwendeten Formeln nämlich, eine statistisch abgeleitete Entropieformel von Miller, Huggins und Flory in Verbindung mit einem quadratischen Ausdruck für die Lösungswärme, gab zwar viele Beobachtungen vorzüglich wieder, nicht aber die Abhängigkeit der kritischen Temperatur vom Polymerisationsgrad. Es wurde vermutet, daß die früher verwandten Fraktionen noch nicht genügend einheitlich seien und hierauf die Diskrepanz zwischen Theorie und Experiment zurückzuführen sei. In der vorliegenden Arbeit wurde besonders großer Wert auf sorgfältige und mehrfache Fraktionierung gelegt. Die beobachteten Löslichkeitskurven mit diesen Präparaten zeigen jedoch, daß der Einfluß einer geringeren oder größe- 